\title{
UN ESTUDIO SOBRE LAS INVESTIGACIONES ACERCA DE LAS IDEAS DE LOS ESTUDIANTES EN FUERZA Y MOVIMIENTO
}

\section{$A$ study on researches about students ideas on force and motion}

\author{
Silvia Giorgi ${ }^{1}$ \\ Sonia Concari \\ Roberto Pozzo
}

\begin{abstract}
Resumo: Se llevó a cabo un análisis exhaustivo de publicaciones sobre preconcepciones o ideas iniciales referidas al tema "cuerpos en movimiento", también comúnmente denominado "fuerza y movimiento". Se estudiaron los aspectos teóricos, metodológicos y conceptuales involucrados en una muestra de investigaciones consistente en 36 trabajos publicados en revistas especializadas y en libros de distintas procedencias y autores. Los sujetos participantes en las investigaciones abarcan distintos niveles y tipos de instrucción. Los resultados revelan, por un lado la existencia de distintos objetivos, diferentes posturas asumidas por los investigadores con relación a las ideas iniciales, fuentes de datos utilizadas variadas, así como heterogeneidad en los resultados obtenidos y en su interpretación. Por otro lado, del estudio realizado surge que si bien los sujetos no poseen una conceptualización científica de fuerza, en las representaciones que ellos construyen de los fenómenos mecánicos podrían estar involucradas significaciones del término Fuerza asociadas a otras magnitudes físicas tales como cantidad de movimiento y energía. Se concluye en la necesidad de abordar el problema de las preconcepciones desde una nueva perspectiva tanto teórica como metodológica.
\end{abstract}

Unitermos: investigaciones en Mecánica, preconcepciones, conceptos físicos, enseñanza, aprendizaje, significados.

Abstract: An exhaustive analysis of publications about human preconceptions or initial ideas in regard to "bodies in movement" was carried out. Theoretical, methodological and conceptual aspects of a sample of 36 works on individuals of diverse levels of instruction and published in journals and books of different origins, were studied. Our findings revealed a great variety of goals as well as of postures assumed by the investigators with respect to preconceptions. Types of data sources as well as results and their interpretation showed also a great dispersion. From another point of view, the results of the present study suggest that although the subjects' conceptualization of force may be wrong, in their representations of mechanics phenomena therewould existed meanings of the word force associated with other physics' concepts such as momentum and energy. We conclude that it is the time for a new approach to the problem of preconceptions in both, theoretical and methodological fields.

Keywords: investigations about Mechanics, preconceptions, physics concepts, teaching, learning, meanings.

\section{Marco Teórico}

Las ideas intuitivas o concepciones de los estudiantes sobre cuerpos en movimiento han sido exhaustivamente investigadas considerándose en cierta manera que el tema estaba prácticamente agotado. Excepcionalmente algunos pocos autores han presentado cuestionamientos de fondo sobre estos estudios.

Jiménez Gómez y otros (1997) en su revisión de trabajos de investigación en preconcepciones en Mecánica que abarcan el periodo 1979 - 1995, encontraron que hay una gran diversidad de objetivos, muestras y técnicas de recogida de datos, indicando que esta heterogeneidad dificulta establecer semejanzas y diferencias en las contribuciones sobre "lo que el

${ }^{1}$ GIDEAF-Departamento de Física - Facultad de Ingeniería Química. - Universidad Nacional del Litoral. - Santiago del Estero-Argentina.E-mail: sgiorgi@fiqus.unl.edu.ar 
alumno sabe". A pesar de que se ofrecen resultados diferentes y especificaciones de carácter metodológico, los autores mencionan que la diversidad en los resultados se debe, más que a una progresión en la metodología o en el marco teórico, a los hechos físicos estudiados, al tipo de preguntas realizadas y a la muestra seleccionada. Notablemente, encuentran que todos los autores (a excepción de Gunstone y White, 1981) utilizan en sus preguntas el término Fuerza. De esto concluyen que las respuestas del alumno estén mediatizadas por la propia pregunta. Así, el sujeto tiene que generar una respuesta sin que se le dé opción a reconsiderar el hecho físico presentado desde una perspectiva diferente a la que se deduce del marco teórico actual de la Mecánica Clásica. Esto podría justificar que los investigadores, al analizar las respuestas de los alumnos a partir del propio contenido objeto de búsqueda, sólo las describan como "correctas" / "incorrectas", o "adecuadas" / "inadecuadas".

El constructivismo (Ausubel y otros, 1991), se fundamenta en el supuesto de que el alumno aprende los contenidos objeto de enseñanza mediante una construcción activa a partir de "lo que sabe". No obstante, Jiménez Gómez y otros $(1994,1997)$ encontraron que las distintas propuestas sobre "lo que el alumno sabe" han estado poco coordinadas. Así, la metodología de investigación no es compartida, los objetivos últimos no están definidos, las implicancias en el aula tampoco han sido coordinadas y la terminología no ha sido consensuada. Estos mismos autores (1997) sostienen que es necesario realizar un estudio profundo acerca de las metodologías y marcos teóricos que subyacen en los trabajos de investigación, para a partir de ahí, intentar hacer confluir lo que otros educadores e investigadores han reclamado: la necesidad de realizar una reflexión teórica, respecto a los paradigmas educativos que han surgido de la filosofía de la ciencia en la tradición del cambio conceptual y de la sicología genética, dando lugar a un nuevo programa de investigación más fructífero y mejor fundamentado teóricamente.

Gilbert, Osborne y Fensham (1982) coincidiendo con McCloskey (1983) señalan, entre otros, dos aspectos importantes a tener en cuenta en la enseñanza de las ciencias. Uno de ellos se relaciona con el lenguaje cotidiano; muchas palabras en ciencia son usadas de una manera alternativa en el lenguaje cotidiano. A menudo un estudiante puede escuchar o leer un enunciado en ciencia y darle sentido usando la interpretación cotidiana de la palabra. Dicha interpretación no es coincidente con la que intenta enseñar el docente o trasmitir el escritor de un libro. El otro aspecto está relacionado conque es frecuente que los estudiantes asignen a los objetos el contenido una cierta cantidad de una magnitud física como por ejemplo fuerza, momentum, energía, etc., en situaciones físicas reales que no pueden justificar. Para algunas magnitudes físicas como por ejemplo fuerza, esta tendencia de los estudiantes conduce a dificultades considerables en el aprendizaje, particularmente en apreciar la naturaleza abstracta de estos conceptos y sus relaciones con otros conceptos.

La presunción de que se pueden usar conceptos expertos como una lente a través de la cual mirar a los conceptos cotidianos es claramente cuestionable. diSessa $(1993,1998)$ sostiene que las ideas de los expertos no pueden ser el único punto de referencia, y que si aún no se ha conseguido una posición para identificar los conceptos expertos, no debemos cometer el error de presumir que existe un alineamiento simple entre los conceptos de los expertos (entendidos psicológicamente) y el vocabulario técnico del dominio. Si se busca una teoría de cambio conceptual, necesitamos ser capaces de describir los conceptos de los novicios y de los expertos en sus propios términos. Debemos describir en detalle qué hacen los estudiantes, en lugar de listar lo que no hacen. Por otro lado, Viennot (1995) indica que la referencia usada para evaluar al conocimiento previo ha sido la Física "correcta" y sugiere que esta referencia "obvia" debería reverse críticamente. A pesar de que la Física "correcta" es una buena base para contrastar las resistentes ideas previas de los estudiantes, este hecho no debería impedir que las 
Un estudio sobre las investigaciones...

investigaciones se orientaran hacia la búsqueda de nuevas maneras de organizar los resultados. Estas nuevas perspectivas de cambio, deberían sugerir una mirada a la Física a través de una nueva óptica. Viennot sostiene como idea principal revisar los contenidos de la Física, y tratar de describir las ideas de los estudiantes y las formas de razonamiento, señalando que estas son dos actividades de aprovechamiento mutuo.

En el presente trabajo se aborda el análisis de los aspectos teóricos, metodológicos y conceptuales involucrados en las investigaciones sobre las preconcepciones o ideas iniciales, sobre la base de la hipótesis de que dichas investigaciones referidas al tema "cuerpos en movimiento" están sesgadas, en general, por una tendencia a identificar semánticamente las palabras que designan conceptos físicos usadas por los sujetos participantes, con tales conceptos científicos.

\section{Metodología}

La muestra de investigaciones analizadas está conformada por 36 trabajos publicados en un amplio espectro de revistas especializadas y en libros de distintas procedencias, y realizados sobre sujetos participantes de variadas culturas e idiomas. El listado de los mismos se muestra en el Anexo. Se empleó como técnica para el estudio, el análisis de contenido (Ander Egg, 1995). A partir de un proceso de categorización previa, llevado a cabo sobre la base de las variables de interés definidas, se analizaron textualmente los trabajos identificando las dimensiones de análisis relevantes que se detallan más adelante.

Se consideraron relevantes las siguientes variables: Tipos de posturas del investigador con relación a las ideas iniciales (V1), Tipos de objetivos planteados por los investigadores (V2), Niveles y tipos de instrucción de los sujetos involucrados en las investigaciones (V3), Tipos de fuentes de datos (V4), Tipos de planteos de las situaciones problemáticas y/o de las consignas involucradas en las tareas propuestas (V5), y Aspectos relevantes relacionados con los resultados informados en la muestra de trabajos analizada y su interpretación (V6).

\section{Análisis e interpretación de los resultados}

\section{V1. Tipos de posturas del investigador con relación a las ideas iniciales}

Las posturas adoptadas por los autores con relación a las ideas iniciales se enmarcan según dos criterios:

1. Las ideas iniciales necesitan ser reemplazadas, sustituidas o eliminadas: para quienes se ubican en esta postura, las ideas iniciales son erróneas, y obstaculizan el aprendizaje, por lo que las mismas deben ser eliminadas, reemplazadas o sustituidas por las ideas científicas correctas, aprendizaje mediante.

2. Las ideas iniciales necesitan ser modificadas, remodeladas, transformadas, integradas $o$ adaptadas: los autores que sostienen esta postura, más acorde con el constructivismo, consideran que las ideas iniciales de los sujetos en el proceso de aprendizaje deben ser respetadas y no siempre son erróneas. Las mismas deberían ser consideradas como el punto de partida para la construcción del conocimiento.

Se encontró que no existe preferencia hacia alguna de las posturas señaladas: ambas se reparten entre las publicaciones investigadas con frecuencias semejantes. En la Tabla 1 se informa con la clave numérica correspondiente (1 ó 2) la postura adoptada por cada autor. 


\section{V2. Tipos de objetivos planteados por los investigadores}

Se identificaron cinco tipos de objetivos diferenciados planteados por los autores. Ellos son: 1. Evaluar la efectividad en la enseñanza; 2. Caracterizar las ideas iniciales o preconcepciones; 3. Investigar sobre aspectos relativos al proceso de aprendizaje; 4 . Estudiar acerca de la comprensión de conceptos y leyes; 5 . Desarrollar una teoría sobre las preconcepciones.

En 7 de las 36 publicaciones los autores plantean estudiar aspectos relacionados con la efectividad en la enseñanza, mientras que en 18 de las mismas se abordan objetivos relacionados con la caracterización de las ideas iniciales. En 13 trabajos los objetivos están relacionados con la comprensión de conceptos y leyes, y en 4 de los mismos el interés se centra en el proceso de aprendizaje. Sólo en 2 de los trabajos se intenta el desarrollo de una teoría acerca de las preconcepciones. En la Tabla 1 se consignan los tipos de objetivos (1, 2, 3, 4 y/o 5) planteados por cada autor.

Cabe señalar que algunos autores plantean más de un tipo de objetivo. No obstante, en la mayoría de esas publicaciones los objetivos se relacionan con la caracterización de las ideas iniciales. Algunos de los autores de estos trabajos acotan sus investigaciones planteándolas en el marco de posibles correspondencias entre las ideas iniciales de los estudiantes e ideas que forman parte de teorías históricamente superadas, consideradas actualmente erróneas. Se indican con $2^{*}$ en la Tabla 1.

\section{V3. Niveles y tipos de instrucción de los sujetos involucrados en las investigaciones}

Se encontró que las investigaciones analizadas fueron realizadas sobre un amplio espectro tanto en nivel (universitario a inicial) como en tipo de instrucción (maestros, estudiantes de profesorado, adultos no científicos).

En 22 de las 36 investigaciones participaron estudiantes universitarios. En 28 de las mismas los estudiantes fueron de escuela secundaria. En 2 de los trabajos se informa que los sujetos correspondían a alumnos de escuela primaria. En 3 intervinieron estudiantes de profesorado. Sólo en una de las publicaciones analizadas se trabajó con maestros y en otra con adultos no científicos. Como surge de las frecuencias absolutas señaladas, los sujetos más frecuentemente involucrados en los trabajos analizados fueron estudiantes de escuela secundaria y universitarios.

Un número considerable de autores trabajó con estudiantes de distintos niveles de instrucción, mientras que otros trabajaron con participantes de distintos niveles y tipos de instrucción. En la Tabla 1 se detallan los niveles y tipos de instrucción de los sujetos con los que se trabajó en cada investigación de acuerdo a la siguiente clave numérica representativa de las distintas categorías: 1. Estudiantes universitarios; 2. Estudiantes de escuela secundaria; 3. Estudiantes de escuela primaria; 4. Estudiantes de profesorado; 5 . Maestros; 6 . Adultos no científicos.

\section{V4. Tipos de fuentes de datos}

Varias fueron las fuentes de datos utilizadas en las distintas investigaciones analizadas: 1 . Transcripciones de entrevistas sobre situaciones experimentales; 2 . Transcripciones de entrevistas sobre situaciones no experimentales; 3 . Respuestas a cuestionarios y/o problemas dadas en forma escrita; 4 . Informes escritos acerca de situaciones experimentales confeccionados por los sujetos; 5 . Registros de observaciones de actividades experimentales abordadas por los sujetos; 6. Registros de observaciones de actividades realizadas por los sujetos (actividades de clases, al contestar las preguntas etc.); 7 . Transcripciones de grabaciones de discusiones de los sujetos en actividades áulicas (trabajos grupales con el uso de simulaciones, resolución de problemas etc.); 8. Pruebas escritas de selección múltiple. 
Las fuentes de datos más frecuentemente usadas fueron las respuestas dadas por los sujetos a cuestionarios y/o resolución de problemas en forma escrita (16/36 publicaciones), y a pruebas escritas de selección múltiple (16/36 publicaciones). Se analizaron transcripciones de entrevistas sobre situaciones experimentales en sólo 3 trabajos, mientras que se encontró un número considerable de los mismos (13) en los que se recurrió a transcripciones sobre situaciones no experimentales. En igual número de publicaciones (2) se usaron informes escritos sobre situaciones experimentales, registros de observaciones de actividades realizadas por los estudiantes y transcripciones de grabaciones de discusiones de los mismos en actividades áulicas. Sólo en una publicación se apeló a registros de observaciones de actividades experimentales de laboratorio. Las fuentes de datos $(1,2,3,4,5,6,7$ y/u 8) utilizadas en cada trabajo se informan en la Tabla 1.

En algunos trabajos los propios autores cuestionan los instrumentos usados para la recolección de información. Clement y otros (1989) señalan que usar solamente una prueba de selección múltiple no garantiza que los sujetos que responden correctamente posean las mismas concepciones de anclaje que hay en la mente del investigador. Otros autores como Guidoni y otros (1995), Montanero y otros (1995) Palmer (1997), Sebastiá (1984) y Thijs (1992) mencionan que el uso de cuestionarios de selección múltiple tiene limitaciones. Estos investigadores detectaron que frecuentemente los sujetos seleccionan la respuesta correcta, pero dan explicaciones incorrectas acerca de su elección, los mismos sugieren que en la evaluación de las respuestas se debe poner más énfasis en los comentarios realizados por los sujetos.

V5. Tipos de planteos de las situaciones problemáticas y/o de las consignas involucradas en las tareas propuestas

El análisis de esta variable se focalizó en determinar si en el planteo de las situaciones problemáticas y/o en las consignas de las tareas propuestas a los participantes: 1. Aparece explícitamente la palabra Fuerza; 2. No aparece explícitamente la palabra Fuerza.

Es notable que en 32 de las 36 publicaciones analizadas se mencione explícitamente la palabra Fuerza en dichas instancias, mientras que sólo en 3 de las mismas no se hace referencia a dicho concepto. En uno de los trabajos los autores no explicitan el tipo de preguntas realizadas. La categoría ( 1 ó 2 ) correspondiente a cada trabajo se detalla en la Tabla 1. 
Tabla 1: Dimensiones de cada una de las variables para las publicaciones analizadas ${ }^{I}$

\begin{tabular}{|c|c|c|c|c|c|c|c|}
\hline \multirow{2}{*}{ Trabajo } & \multirow{2}{*}{ V1 } & \multirow{2}{*}{$\mathrm{V} 2$} & \multirow{2}{*}{ V3 } & \multirow{2}{*}{ V4 } & \multirow{2}{*}{ V5 } & \multicolumn{2}{|r|}{ V6 } \\
\hline & & & & & & 6.1 & 6.2 \\
\hline Acevedo Díaz (1989) & 1 & 1 & 2 & 3 & 2 & & \\
\hline Boeha (1990) & & $2^{*}$ & 2 & 2,3 & 1 & 3,4 & $1,3,5$ \\
\hline Champagne y otros (1980) & 1 & 2 & 1 & 4 & & & 2,6 \\
\hline Clement (1982) & 2 & 2 & 1 & 3,6 & 1 & 2 & 1,2 \\
\hline Clement y otros (1989) & 2 & 2 & 2 & 8 & 1 & & \\
\hline diSessa (1993) & 2 & 5 & $1,2,6$ & 2 & 1 & $2,3,4$ & $1,2,3,5$ \\
\hline diSessa (1998) & 2 & 4,5 & 1 & 1 & 1 & & 1,2 \\
\hline Finegold y Gorsky (1991) & 1 & 2,4 & 1,2 & 2,3 & 1 & 2,4 & 1,3 \\
\hline Galili y Bar (1992) & 1 & 1 & $1,2,4$ & 3 & 1 & 2 & $1,2,3$ \\
\hline Gilbert y otros (1982) & 2 & 2 & 2 & 1,2 & 1 & 1 & 3 \\
\hline Guidoni y otros (1995) & 1 & 3 & 1 & 8 & 1 & & 1,2 \\
\hline Gunstone y White (1981) & 2 & 4 & 1 & 5,8 & 2 & 1 & \\
\hline Halloun y Hestenes (1985a) & 2 & 1 & 1,2 & 8 & 1 & & \\
\hline Halloun y Hestenes (1985b) & 2 & $2^{*}$ & 1 & 2,8 & 1 & 1,2 & $1,2,3,4,5,6,7$ \\
\hline Hestenes y otros $(1992 \mathrm{a})$ & 1 & 1,4 & 1,2 & 8 & 1 & 1,2 & $1,2,3,4,5,6,7$ \\
\hline Hestenes y otros (1992b) & 1 & 1,4 & 1,2 & 8 & 1 & & \\
\hline Kuiper (1994) & 1 & 4 & 2 & 8 & 1 & 2 & $1,3,6$ \\
\hline McCloskey (1983) & 1 & 2 & 1,2 & 2,3 & 2 & 1,2 & 5 \\
\hline Mohapatra (1989) & 2 & 4 & 2,4 & 2,3 & 1 & 1 & 2,6 \\
\hline Montanero y otros (1995) & 1 & 4 & 1,2 & 2,8 & 1 & 3 & $1,2,3,4,7$ \\
\hline Moraes y Moraes (2000) & & 4 & 1,2 & 8 & 1 & & 2 \\
\hline Oliva (2001) & 2 & 3 & 2 & 3 & 1 & 2 & $1,3,4,7$ \\
\hline Osborne (1991) & 2 & 2 & $1,2,3$ & 2 & 1 & 3 & $1,3,5,6,7$ \\
\hline Palmer (1997) & 1 & 2 & 1,2 & 8 & 1 & & $1,3,4$ \\
\hline Sebastiá (1984) & 1 & $2^{*}$ & 1,2 & 8 & 1 & & $1,3,7$ \\
\hline Tao y Gunstone (1999) & & 2 & 2 & $2,3,7,8$ & 1 & & $1,2,3,5,6$ \\
\hline Thijs (1992) & 1 & 1 & 2 & $3,4,6,7,8$ & 1 & 3 & $1,2,3,6,7$ \\
\hline Thornton (1995) & 1 & $2,3,4$ & 1,2 & 8 & 1 & 3,4 & $1,2,4,6$ \\
\hline Trumper y Gorsky (1996) & 2 & $2 *, 4$ & 4 & 3 & 1 & 2 & $1,3,4,5,6,7$ \\
\hline Twigger y otros (1994) & 2 & 2 & 2 & 2,3 & 1 & 3,4 & $1,2,3,5$ \\
\hline Viennot (1979) & 1 & 1,2 & 1,2 & 3 & 1 & 2,4 & $1,2,3,6,7$ \\
\hline Villani y Orquiza (1997) & 2 & 3 & 2 & 1,3 & 1 & 1 & 1,7 \\
\hline Watts (1983) & 2 & 4 & 1,2 & 2 & 1 & 3,4 & $1,2,3,4,5,6,7$ \\
\hline Watts y Zylbersztajn (1981) & 2 & 2 & 2,5 & 2,8 & 1 & 3,4 & $1,2,3,4,7$ \\
\hline White (1983) & 1 & 4 & 2,3 & 3 & 1 & & \\
\hline Whiteley (1994) & & 2 & 1 & 3 & 1 & 1 & $1,2,5,6$ \\
\hline
\end{tabular}

${ }^{1}$ Los números corresponden a las dimensiones de cada variable $V_{\mathrm{i}}$.

V6. Aspectos relevantes relacionados con los resultados informados en la muestra de trabajos analizada y su interpretación

En el análisis de los trabajos se pudieron distinguir distintas afirmaciones hechas por los autores acerca de las características del pensamiento de los sujetos. También se identificaron algunos aspectos de dicho pensamiento informados por los autores en sus resultados y en su interpretación, que resultaron relevantes para esta investigación. A continuación se presentan dichos resultados relacionados con cada uno de esos aspectos. 


\section{V6.1 Uso de las Palabras Coincidentes con las Denominaciones de Conceptos Físicos (PCDCF) con un significado diferente al cientifico}

Se identificaron las siguientes afirmaciones de los autores: 1. Los sujetos usan las PCDCF con un significado diferente al científico; 2. Los sujetos usan la palabra Fuerza asociada a la idea de ímpetus; 3. Los sujetos usan la palabra Fuerza asociada al significado de la magnitud física cantidad de movimiento (CM); y/o 4. Los sujetos usan la palabra Fuerza asociada al significado de energía. Así por ejemplo, algunos autores hablan sólo de una confusión entre magnitudes físicas (categoría 1), mientras que otros establecen una correspondencia específica entre Fuerza y otras ideas o conceptos (categorías 2, 3 y/o 4). Otros autores hacen afirmaciones involucrando las categorías 1 y 2 , y/o 3 , y/o 4.

En 24 de las 36 publicaciones se informó que los sujetos confunden magnitudes físicas como ser velocidad, aceleración, fuerza, potencia y energía, o se mencionó correspondencia del uso de la palabra Fuerza con la idea de ímpetus, o con los conceptos físicos de CM y/o energía. En algo menos de una cuarta parte de los trabajos se informa que los sujetos confunden magnitudes físicas, pero sin emitir juicio acerca de los posibles significados que pueden atribuirse a esas palabras en el contexto de uso de las mismas por parte de los sujetos. En 11 trabajos se mencionó correspondencia entre el significado de uso de la palabra Fuerza y la idea de ímpetus, calificando las ideas de los sujetos como enmarcadas en teorías científicas históricamente superadas. En 9 publicaciones se señala alguna correspondencia entre el significado de uso de la palabra Fuerza y el concepto físico de CM, mientras que en 8 de las mismas la correspondencia se establece con el concepto de energía.

Se investigó también la posible correspondencia entre las distintas posturas adoptadas por los autores y los significados de uso por parte de los sujetos de la palabra Fuerza, de acuerdo a lo informado en los resultados de las investigaciones. No se encontró relación entre la diversidad de resultados informados y dichas posturas.

\section{V6.2 Tipos de correspondencias informados por los autores de los trabajos analizados} entre el significado de uso de la palabra Fuerza, y aspectos de las ideas intuitivas considerados relevantes en el presente estudio

Se consideraron relevantes en los resultados de las publicaciones los siguientes aspectos de las ideas intuitivas asociados a la palabra Fuerza: 1. La asociación necesaria entre fuerza y dirección del movimiento; 2. La relación directa de Fuerza con velocidad en cuerpos en movimiento; 3. Los cuerpos en movimiento poseen fuerza interna o están dotados de fuerza; 4. Los cuerpos en movimiento de mayor masa, o que se mueven a mayor velocidad, ejercen o poseen la mayor fuerza; 5. La "fuerza de movimiento" contenida en los cuerpos se consume, se pierde o disipa durante el movimiento; 6 . En los cuerpos en reposo no actúan fuerzas; 7. La "fuerza de movimiento" se transmite a otros cuerpos (por contacto o a través de un medio). En la Tabla 2 se sintetizan los resultados del análisis de correspondencia entre los aspectos de las ideas intuitivas considerados relevantes para este estudio (variable V6.2), y los tipos de asociación del uso de la palabra Fuerza con otro significado (dimensiones 2, 3 y 4 de la variable V6.1).

De la lectura de la tabla surge que, teniendo en cuenta las ideas intuitivas en las que los sujetos asocian una fuerza en la dirección del movimiento y una fuerza interna contenida o poseída por los cuerpos en movimiento, las mayores frecuencias de ocurrencia se dieron en trabajos donde se informa de las correspondencias de Fuerza con ímpetus y con el concepto de $\mathrm{CM}$. En cuanto a la concepción de fuerza en relación directa con la velocidad de cuerpos en movimiento se observa que la mayor incidencia, en cuanto a número de trabajos, se da en aquellos 
en que se manifiesta la correspondencia de Fuerza con CM y de no - correspondencia con ningún otro concepto o idea.

En los casos en que los sujetos consideran que los cuerpos en movimiento de mayor masa, o que se mueven a mayor velocidad, ejercen o poseen la mayor fuerza; o en los que consideran que en los cuerpos en movimiento la "fuerza de movimiento" se consume, se pierde o disipa; o que sobre los cuerpos en reposo no actúan fuerzas; y que "la fuerza de movimiento" se transmite a otros cuerpos (por contacto o a través de un medio), se observa que los mayores números de trabajos se dieron en la categoría que contempla el uso del término Fuerza asociado a la idea de ímpetus.

\section{Cconclusiones}

Surge como conclusión primaria de este análisis, que las significaciones involucradas en las ideas de los sujetos relacionadas con el término Fuerza, pueden estar asociadas a distintas magnitudes físicas tales como CM y energía. De acuerdo con las interpretaciones de los resultados de las publicaciones que informan que los sujetos usan la palabra Fuerza con un significado cercano a la idea de ímpetus, y/o a CM, y/o energía se desprende que, aunque los sujetos no usan la palabra Fuerza con su significado científico, las ideas relacionadas con ese término pueden estar próximas a los significados de otros conceptos científicos. Enfocando el problema de las preconcepciones desde un nuevo ángulo, se podría decir que en las ideas iniciales de los sujetos sobre cuerpos en movimiento existen concepciones cercanas a conceptos físicos tales como CM y energía, pero los mismos son exteriorizados empleando la palabra Fuerza. Desde esta perspectiva el problema de las ideas intuitivas estaría relacionado con cuestiones semánticas, basándose fundamentalmente en una cuestión de comunicación e interpretación.

Los resultados de esta investigación muestran que las significaciones involucradas en las ideas de los sujetos relacionadas con el término Fuerza, pueden estar asociadas a distintas magnitudes físicas tales como CM y energía. De acuerdo con las interpretaciones de los resultados de las publicaciones que informan que los sujetos usan la palabra Fuerza con un significado cercano a la idea de ímpetus, y/o a CM, y/o energía, surge que aunque los sujetos no usan la palabra Fuerza con su significado científico, las ideas relacionadas con ese término pueden estar próximas a los significados de otros conceptos científicos.

Del estudio realizado, se observa que no hay relación entre la diversidad de resultados informados, y los aspectos considerados de las ideas intuitivas de los estudiantes, asociados a la palabra Fuerza. Además, aunque la mayoría de los autores identifican más frecuentemente tres de los aspectos de las ideas intuitivas de los sujetos: fuerza en la dirección del movimiento, fuerza en relación directa con la velocidad y fuerza interna en cuerpos en movimiento, características todas propias de la magnitud cantidad de movimiento en sólo una cuarta parte de las publicaciones analizadas se considera la posibilidad de que cuando los sujetos hablan de fuerza, estén en realidad refiriéndose (sin saberlo) a la magnitud CM. 
Un estudio sobre las investigaciones...

Tabla 2: Frecuencia absoluta de publicaciones en las que se corresponden los aspectos de las ideas intuitivas relevantes para este estudio con tipos de asociación de uso de la palabra Fuerza con otro significado

\begin{tabular}{|c|c|c|c|c|}
\hline \multirow[b]{2}{*}{ Aspectos de las ideas intuitivas: } & \multicolumn{3}{|c|}{ Fuerza asociada con: } & \multirow{2}{*}{$\begin{array}{l}\text { Ningún tipo de } \\
\text { asociación }\end{array}$} \\
\hline & $\mathrm{CM}$ & energía & ímpetus & \\
\hline $\begin{array}{l}\text { 1. Asociación necesaria entre fuerza y dirección del } \\
\text { movimiento } \\
25 / 36\end{array}$ & 9 & 8 & 10 & 7 \\
\hline $\begin{array}{l}\text { 2. Relación directa de Fuerza con velocidad en cuerpos } \\
\text { en movimiento } \\
19 / 36\end{array}$ & 7 & 6 & 6 & 7 \\
\hline $\begin{array}{l}\text { 3. Los cuerpos en movimiento poseen fuerza interna o } \\
\text { están dotados de fuerza } \\
\qquad 20 / 36\end{array}$ & 8 & 7 & 9 & 4 \\
\hline $\begin{array}{l}\text { 4. Los cuerpos en movimiento de mayor masa, o que se } \\
\text { mueven a mayor velocidad, ejercen o poseen la mayor } \\
\text { fuerza } \\
\qquad / 36\end{array}$ & 1 & 3 & 4 & 1 \\
\hline $\begin{array}{l}\text { 5. La "fuerza de movimiento" se consume, pierde o } \\
\text { disipa } \\
11 / 36\end{array}$ & 2 & 4 & 5 & 2 \\
\hline $\begin{array}{l}\text { 6. En los cuerpos en reposo no actúan fuerzas } \\
13 / 36\end{array}$ & 2 & 3 & 5 & 4 \\
\hline $\begin{array}{l}\text { 7. La "fuerza de movimiento" se transmite entre } \\
\text { cuerpos } \\
12 / 36\end{array}$ & 3 & 3 & 5 & 2 \\
\hline
\end{tabular}

Casi la tercera parte de los autores menciona una asociación entre el significado de uso por parte de los sujetos de la palabra Fuerza y la idea de ímpetus, aludiendo de alguna manera a una analogía entre el desarrollo histórico del conocimiento científico y la construcción del conocimiento por parte de los individuos. Si se tiene en cuenta lo anterior, y el hecho de que existen aspectos de las ideas intuitivas de los sujetos que están asociados a la palabra Fuerza y que son propios del concepto de CM, aparece un área a explorar consistente en profundizar cómo este concepto físico subyace en las ideas iniciales de los estudiantes cuando usan la palabra Fuerza.

Con relación a las características de los instrumentos de recolección de información, en casi la totalidad de los trabajos analizados los autores hacen uso explícito del término Fuerza en el planteo de las situaciones problemáticas y/o en las consignas involucradas en las tareas propuestas a los sujetos, de este modo los mismos no son alentados a utilizar para explicar la situación otras palabras y/o conceptos que podrían manejar más cómodamente. Esto contribuye a que no se tenga en cuenta la posibilidad de considerar otras interpretaciones, acotando en gran medida la búsqueda de lo que el "alumno sabe". La cuestión metodológica señalada, sumada a la diversidad de resultados informados, pone en duda la validez de algunas interpretaciones e inferencias realizadas por los investigadores, como ha sido también detectado por Jiménez Gómez y otros (1997). Esto corrobora parcialmente la hipótesis formulada en el presente trabajo.

La investigación en el tema de las preconcepciones en Mecánica lleva ya más de dos décadas, sin embargo, es bien conocido que la eficacia en la enseñanza del tema "cuerpos en movimiento" es muy escasa, las preconcepciones en Mecánica perduran en los sujetos aún después de varios años de enseñanza formal, y hasta en docentes de Física. Pensamos como Jiménez Gómez y otros (op. cit.), que habría que dudar de la eficacia didáctica de los diseños de enseñanza basados en la información que aportó esta línea de investigación ya que los resultados a los que se han llegado están excesivamente mediatizados por la lógica del contenido 
académico objeto de búsqueda. Esos autores señalan que esa ineficacia se debe fundamentalmente a la existencia de importantes diferencias entre el contenido académico y la realidad cognoscitiva del alumno.

Otro resultado relevante de esta investigación es la evidencia de la carencia de una base teórica común sobre las preconcepciones que, como también lo señalaron diSessa (1993, 1998) y Oliva (2001), hace que la comparación de los resultados de las investigaciones sobre el tema resulte dificultosa.

El progreso en el problema de las preconcepciones sería posible si se hiciera una revisión de cuál es la información sobre el alumno que resulte realmente de interés didáctico y a la vez, si se utilizaran contextos teóricos que permitan un mayor acercamiento a la realidad cognoscitiva del que aprende, lo que llevaría necesariamente a un cambio metodológico en la búsqueda de esta información, en coincidencia con lo que Jiménez Gómez y otros (op. cit.) ya han sugerido. Si la búsqueda de "lo que el alumno sabe" se llevara a cabo con metodologías que conlleven a la obtención de información más cercana a las peculiaridades cognoscitivas de los sujetos, los diseños de enseñanza podrían adecuarse más a esas peculiaridades, y consecuentemente se incrementaría la eficacia didáctica.

Por lo expuesto, creemos que los resultados de esta investigación bibliográfica proveen pautas para abordar el problema de las preconcepciones desde otra perspectiva más amplia y menos sesgada, tanto desde el punto de vista teórico como metodológico.

AGRADECIMIENTO

Este trabajo ha sido realizado en el marco de los proyectos CAI+D 2000-UNL, Código: 12/H193 y PICT CONICET 03080.

\section{Referencias}

ANDER EGG, E. Técnicas de investigación social. 24. ed. Buenos Aires: Lumen, 1995.

AUSUBEL, D.; NOVAK, J.; HANESIAN, H. Psicología educacional, un punto de vista cognitivo. México: Trillas, 1991.

JIMÉNEZ GÓMEZ, E.; MARÍN MARTÍNEZ, N. Problemas de terminología en estudios realizados sobre "lo que el alumno sabe" en ciencias. Enseñanza de las Ciencias, Barcelona, v. 12, n. 2, p. 235-245, 1994.

JIMÉNEZ GÓMEZ, E.; SOLANO, L.; MARÍN, N. Estudio de la progresión en la delimitación de las "ideas" del alumno sobre fuerza. Enseñanza de las Ciencias, Barcelona, v. 15, n. 3, p. 309-328, 1997.

VIENNOT, L. The contents of physics. Essential elements, common views. In: BERNARDINI, C. et al. (Ed.). Thinking physics for teaching. New York: Plenum Press, 1995. cap. 5

Nota: Las referencias de los trabajos analizados en esta investigación se listan en el Anexo.

\section{Anexo}

ACEVEDO DÍAZ, J. Comprensión newtoniana de la caída de cuerpos. Un estudio de su evolución en el bachillerato. Enseñanza de las Ciencias, Barcelona, v. 7, n. 3, p. 241-246, 1989. 
BOEHA, B. Aristotle, alive and well in Papua New Guinea science classrooms. Physics Education, London, v. 25, p. 280-283, 1990.

CHAMPAGNE A.; KLOPFER, L.; ANDERSON, J. Factors influencing the learning of classical mechanics. American Journal of Physics, New York, v. 48, n. 12, p. 1074-1079, 1980.

CLEMENT, J. Students' preconceptions in introductory mechanics. American Journal of Physics, New York, v. 50, n. 1, p. 66-71, 1982.

CLEMENT, J.; BROWN, D.; ZIETSMAN, A. Not all preconceptions are misconceptions: finding "anchoring conceptions" for grounding instruction on students' intuitions. International Journal of Science Education, London, v. 11, Especial Issue, p. 554-565, 1989.

DISESSA, A. Toward an Epistemology of Physics. Cognition and Instruction, Mahwah, v. 10, n. 2/3, p. 105-225, 1993.

DISESSA, A. A.; SHERIN, B. What changes in conceptual change? International Journal of Science Education, London, v. 20, n. 10, p. 1155-1191, 1998.

FINEGOLD, M.; GORSKY, P. Students' concepts of force as applied to related physical systems: a search for consistency. International Journal of Science Education, London, v. 13, n. 1, p. 97-113, 1991.

GALILI, I.; BAR, V. Motion implies a force: where to expect vestiges of the misconception?

International Journal of Science Education, London, v. 14, n. 1, p. 63-81, 1992.

GILBERT, J.; OSBORNE, R.; FENSHAM, P. Children's science and its consequences for teaching. Science Education, New York, v. 66, n. 4, p. 623-633, 1982.

GUNSTONE, R.; WHITE, R. Understanding of Gravity. Science Education, New York, v. 65, n. 3, 291-299, 1981.

GUIDONI, P.; PORRO, A.; SASSI, E. Force-motion conceptions. A phenomenological analysis of questionnaires submitted to freshmen physics mayors. In: BERNARDINI, C. et al. (Ed.). Thinking physics for teaching. New York: Plenum Press, 1995. cap. 14.

HALLOUN, I.; HESTENES, D. The initial knowledge state of college physics students. American Journal of Physics, New York, v. 53, n. 11, p. 1043-1055, 1985 a.

HALLOUN, I.; HESTENES, D. Common sense concepts about motion. American Journal of Physics, New York, v. 53, n. 11, p. 1056-1065, 1985 b.

HESTENES, D.; WELLS, M.; SWACKHAMER, G. Force Concept Inventory. Physics Teacher, College Park, v. 30, p. 141-158, 1992a.

HESTENES, D.; WELLS, M.; SWACKHAMER, G. A Mechanics Baseline Test. Physics Teacher, College Park, v. 30, p. 159-166, 1992 b. 
KUIPER, F. Student ideas of science concepts: alternative frameworks? International Journal of Science Education, London, v. 16, n. 3, p. 279-292, 1994.

MCCLOSKEY, M. Naive theories of motion. In: GENTNER, D.; STEVENS, A. L. (Ed.). Mental Models. New Jersey: Lawrence Erlbaum, 1983. cap. 13.

MOHAPATRA, J. Pupils, teachers, induced incorrect generalization and the concept of "force". International Journal of Science Education, London, v. 11, n. 4, p. 429-436, 1989.

MONTANERO, M.; PEREZ, A.; SUERO, M. A survey of students' understanding of colliding bodies. Physics Education, London, v. 30, n. 5, p. 277-283, 1995.

MORAES, A.; MORAES, I. A avaliação conceitual de força e movimento. Revista Brasileira de Ensino de Física, São Paulo, v. 22, n. 2, p. 232-243, 2000.

OLIVA, J. Distintos niveles de análisis para el estudio del cambio conceptual en el dominio de la mecánica. Enseñanza de las Ciencias, Barcelona, v. 19, n. 1, p. 89-102, 2001.

OSBORNE, R. Construir a partir de las ideas intuitivas de los alumnos. In: El aprendizaje de las ciencias: implicaciones de la ciencia de los alumnos. Madrid: Narcea, 1991. cap. 4.

PALMER, D. The effect of context on students' reasoning about forces. International Journal of Science Education, London, v. 19, n. 6, p. 681-696, 1997.

SEBASTIÁ, J. Fuerza y Movimiento: la interpretación de los estudiantes. Enseñanza de las Ciencias, Barcelona, p. 161-169, 1984.

TAO, P.-K.; GUSNTONE, R. The process of conceptual change in force and motion during computersupported physics instruction. Journal of Research in Science Teaching, New York, v. 36, n. 7 , p. 859-882, 1999.

THIJS, G. Evaluation of an introductory course on "force" considering students' preconceptions. Science Education, New York, v. 76, n. 2, p. 155-174, 1992.

THORNTON, R. Conceptual dynamics. Changing student views of force and motion. In: BERNARDINI, C.; TARSITANI, C.; VICENTINI, M. (Ed.). Thinking physics for teaching. New York: Plenum Press, 1995. cap. 13.

TRUMPER, R.; GORSKY, P. A cross-college age study about physics students' conceptions of force in pre-service training for high school teachers. Physics Education, London, v. 31, n. 4, p. 227-236, 1996.

TWIGGER, D. et al. The conception of force and motion of students aged between 10 and 15 years: an interview study designed to guide instruction. International Journal of Science Education, London, v. 16, n. 2, p. 215-229, 1994. 
VIENNOT, L. Spontaneous Reasoning in Elementary Dynamics. European Journal of Science Education, London, v. 1, n. 2, p. 205-221, 1979.

VILLANI, A.; ORQUIZA DE CARVALHO, L. Evolución de las representaciones mentales sobre colisiones. Enseñanza de las Ciencias, Barcelona, v. 15, n. 1, p. 91-102, 1997.

WATTS, M. A study of schoolchildren's alternative frameworks of the concept of force. European Journal of Science Education, London, v. 5, n. 2, p. 217-230, 1983.

WATTS, M.; ZYLBERSZTAJN, A. A survey of some children's ideas about forces. Physics Education, London, v. 16, n. 6, p. 360-365, 1981.

WHITE, B. Sources of difficulty in understanding Newtonian dynamics. Cognitive Science, Norwood, v. 7, n. 41-65, 1983.

WHITELEY, P. Using free body diagrams as a diagnostic instrument. Physics Education, London, v. 31, n. 5, p. 309-313, 1994 . 
